\title{
A SILICON OPTOMECHANICAL ACCELEROMETER WITH HIGH BANDWIDTH AND SENSITIVITY
}

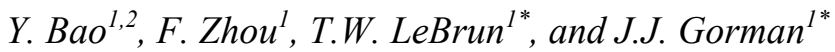 \\ ${ }^{1}$ National Institute of Standards and Technology, Gaithersburg, MD, USA \\ ${ }^{2}$ Theiss Research, La Jolla, CA, USA
}

\begin{abstract}
Accelerometers with high sensitivity and bandwidth are required for numerous critical applications, including structural testing of buildings and bridges, seismic testing for oil and gas exploration, and aerospace systems. This paper presents a new optomechanical accelerometer design for precision vibration measurements that uses a hemispherical optical microcavity to transduce the motion of a proof mass. Experimental testing of the accelerometer shows that this design is capable of resolution below $147 \mu \mathrm{m} / \mathrm{s}^{2} / \sqrt{ } \mathrm{Hz}(15 \mu \mathrm{g} / \sqrt{ } \mathrm{Hz})$ between $725 \mathrm{~Hz}$ and nearly $10 \mathrm{kHz}$.
\end{abstract}

\section{INTRODUCTION}

Fabry-Pérot interferometry is known to be one of the most sensitive approaches for measuring displacement, making it an attractive transduction solution for accelerometers. It has been used in accelerometers previously with several different optical cavity geometries, including an out-of-plane cavity design [1], and in-plane cavity designs with waveguides [2] and optical fibers [3,4]. This paper reports on the design, operation, and first demonstration of an optomechanical accelerometer that uses a silicon hemispherical Fabry-Pérot microcavity to transduce the motion of the accelerometer's proof mass. Our focus here is on achieving both high bandwidth and sensitivity for vibration applications rather than sensors for inertial navigation.

\section{ACCELEROMETER DESIGN AND OPERATION}

The optomechanical accelerometer design is shown in Fig. 1. Two silicon chips are assembled together with a spacer between them to form an optical microcavity. One chip has a concave silicon micromirror [5] and the other has a $10 \mathrm{mg}$ proof mass $(3 \mathrm{~mm} \times 3$ $\mathrm{mm} \times 0.5 \mathrm{~mm}$ ) that is suspended with microscale silicon nitride beams. This design combines a highly stable hemispherical cavity, unlike the plane-parallel cavities used in [1,2], with a wafer-scale microfabrication process, unlike the accelerometers described in $[3,4]$. The assembled chips are packaged with an optical fiber and mode matching lens that couple light in and out of the microcavity. The reflected optical signal is measured using a circulator, photodetector, data acquisition system, and signal analyzer.

The optical spectrum of the microcavity is shown in Fig. 2, which is found by scanning the laser wavelength while measuring the reflected intensity. In this case, a low-finesse optical cavity (i.e., a cavity with high optical loss) is used to generate a broad optical resonance for measuring displacement. The proof mass displacement is measured by positioning a stable laser at the point of highest slope on one of the optical resonances. When the proof mass moves, the optical resonances will shift in wavelength, generating a change in the reflected intensity. The displacement can then be determined from the equation in Fig. 2. A low-finesse cavity is used here because laser locking to the cavity resonance is not required, resulting in a simpler readout approach. However, we are also pursuing accelerometers with high-finesse cavities to maximize the sensitivity.

\section{EXPERIMENTAL RESULTS}

The accelerometer was first tested on a commercial shaker using a swept-sine excitation (Fig. 3). The fundamental resonance

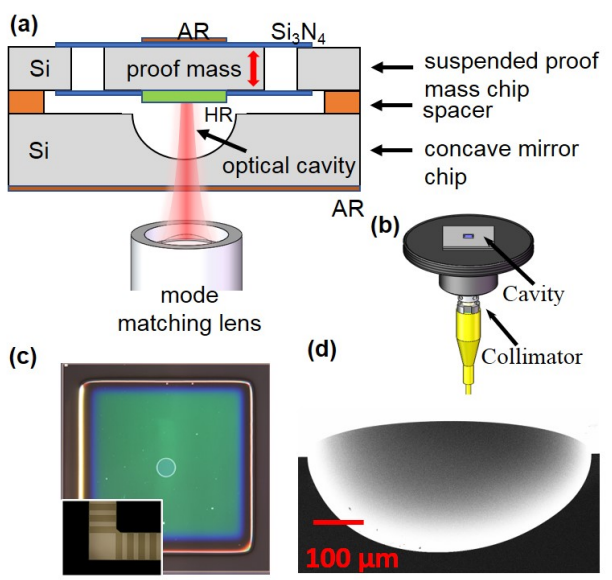

Figure 1: The optomechanical accelerometer design. (a) Crosssectional schematic, AR: Anti-reflection coating, HR: mirror coating. (b) Packaging for the fiber coupled accelerometer. (c) and (d) Images of a fabricated suspended proof mass (3 $\mathrm{mm}$ square) and a silicon concave mirror, respectively.

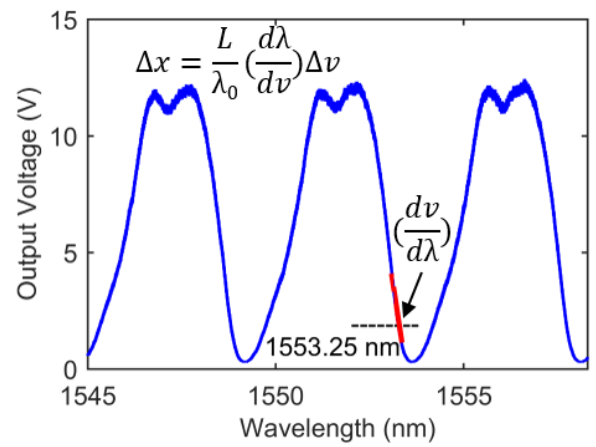

Figure 2. Optical spectrum of the Fabry-Pérot microcavity when measured in reflection with a tunable wavelength laser. Displacement sensing is performed with a fixed laser wavelength that is selected to provide the highest sensitivity (i.e., where the slope is greatest, $\approx 1553.25 \mathrm{~nm}$ ). The measured voltage is converted to displacement using the included equation, where $\lambda_{0}$ is the operating wavelength and $L$ is the length of the microcavity, as determined from the microcavity's free spectral range.

of the suspended proof mass is at $9.6 \mathrm{kHz}$, and a second resonance in the accelerometer package is also seen at lower frequency. We are currently redesigning the accelerometer package to achieve a purer frequency response. The linearity of the accelerometer was also investigated on the shaker, as shown for a $2 \mathrm{kHz}$ excitation in Fig. 4. The accelerometer is clearly linear within the test range, which ran from the noise floor up to about $180 \mathrm{~mm} / \mathrm{s}^{2}$. The relationship between the input acceleration and the displacement of the proof mass at $2 \mathrm{kHz}$ was found to be $0.143 \mathrm{~nm} / \mathrm{m} / \mathrm{s}^{2}(1.4 \mathrm{~nm} / \mathrm{g})$.

The displacement noise spectrum was measured without excitation and with vibration and acoustic isolation to determine the acceleration resolution. The measured displacement noise is shown 

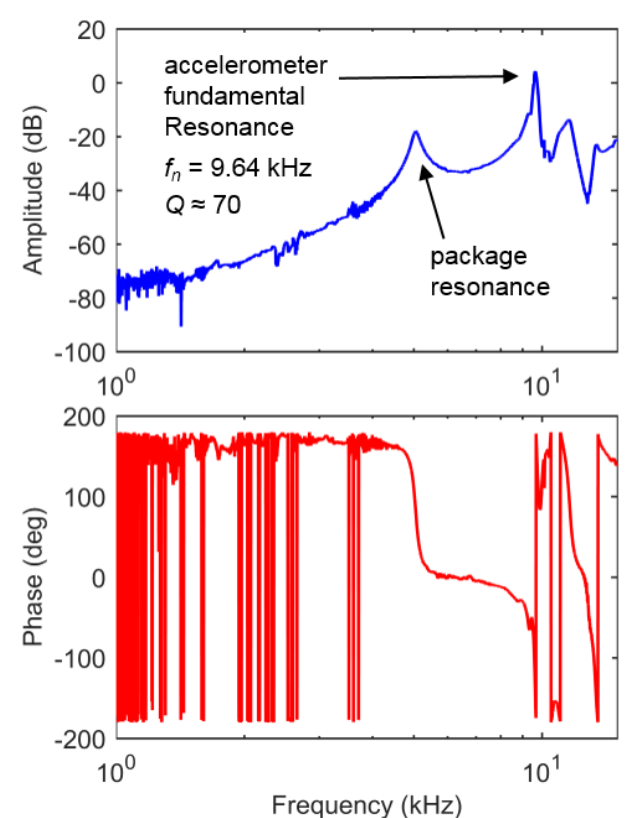

Figure 3. Frequency response of the accelerometer when measured on a commercial shaker with a swept-sine excitation.

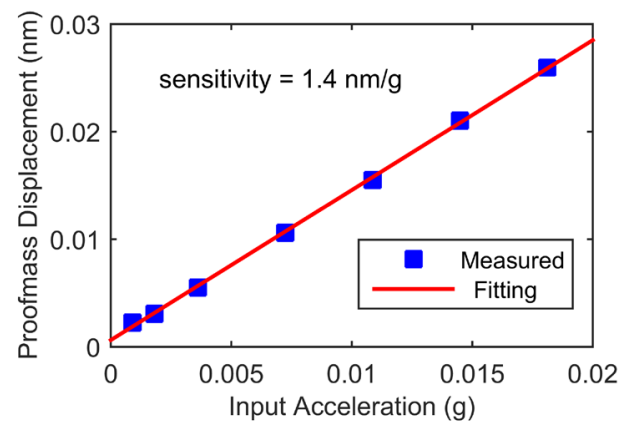

Figure 4. Accelerometer linearity test at $2 \mathrm{kHz}$. The acceleration provided by the shaker was varied and the proof mass displacement was measured with the microcavity. The accelerometer is highly linear from the noise floor to at least $180 \mathrm{~mm} / \mathrm{s}^{2}(\approx 18 \mathrm{mg}) .1 \mathrm{~g}=$ $9.81 \mathrm{~m} / \mathrm{s}^{2}$

in Fig. 5, where the noise floor is $35 \mathrm{fm} / \sqrt{ } \mathrm{Hz}$. This is only about a factor of two higher than the thermomechanical noise of the proof mass on resonance. The suspended proof mass was designed to behave like a simple harmonic oscillator, with all other vibration modes located at significantly higher frequencies by design [6]. In this case, the acceleration noise can be estimated by multiplying the displacement noise by the inverted harmonic oscillator model [3], resulting in Fig. 6. The acceleration noise is below $147 \mu \mathrm{m} / \mathrm{s}^{2} / \sqrt{\mathrm{Hz}}$ $(15 \mu \mathrm{g} / \sqrt{\mathrm{Hz}})$ beyond $725 \mathrm{~Hz}$. This sensitivity and bandwidth performance is better than reported in $[1,2]$ and equivalent to that found in [4], while also providing a more scalable micromechanical design and fabrication process. Since the first resonance is $9.6 \mathrm{kHz}$, the accelerometer has considerable bandwidth while also achieving near micro- $g$ resolution, making it well suited for precision vibration measurements at high frequency.

\section{ACKNOWLEDGMENTS}

Research performed in part in the NIST Center for Nanoscale Science and Technology Nanofab.

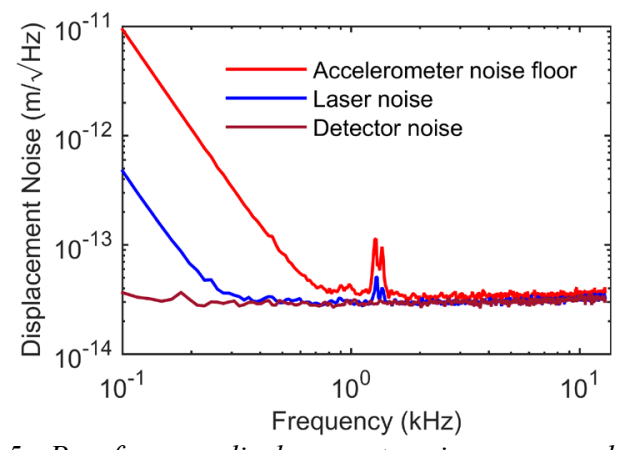

Figure 5. Proof mass displacement noise measured with the microcavity readout when the external acceleration is zero and the accelerometer is isolated from vibration and acoustic noise.

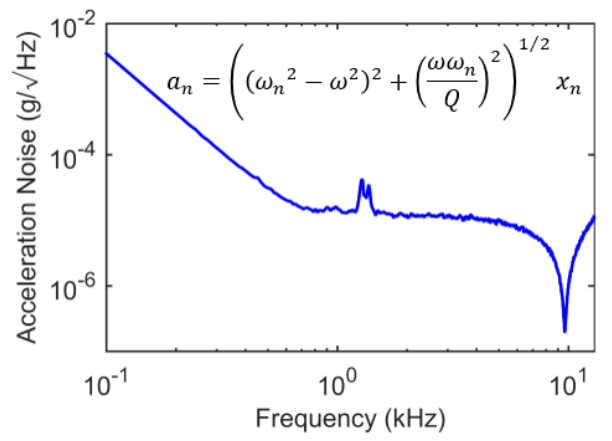

Figure 6. Estimated acceleration noise, $a_{n}$, based on the displacement noise and frequency response of the suspended proof mass. The accelerometer is assumed to behave as a simple harmonic oscillator. Using the equation in the figure, the displacement noise, $x_{n}$, is converted to $a_{n}$ [3], where $\omega_{n}$ is the natural frequency, $Q$ is the quality factor, and $\omega$ is the input frequency. For frequencies above $725 \mathrm{~Hz}$, the noise floor is typically better than $147 \mu \mathrm{m} / \mathrm{s}^{2} / \mathrm{NHz}(15$ $\mu \mathrm{g} / \sqrt{ } \mathrm{Hz})$. The low-frequency response is dominated by $1 / f$ noise in the laser, which can be improved through better laser stabilization. $1 \mathrm{~g}=9.81 \mathrm{~m} / \mathrm{s}^{2}$

\section{REFERENCES}

[1] M.A. Perez and A.M. Shkel, "Design and Demonstration of a Bulk Micromachined Fabry-Pérot $\mu \mathrm{g}$-Resolution Accelerometer", IEEE Sensors Journal, 7, 1653 (2007).

[2] K. Zandi, J.A. Bélanger, and Y.-A. Peter, "Design and Demonstration of an In-Plane Silicon-on-Insulator Optical MEMS Fabry-Pérot-Based Accelerometer Integrated with Channel Waveguides", Journal of Microelectromechanical Systems, 21, 1464 (2012).

[3] F. Guzmán Cervantes et al., "High Sensitivity Optomechanical Reference Accelerometer Over 10 kHz", Applied Physics Letters, 104, 221111 (2014).

[4] O. Gerberding et al., "Optomechanical reference accelerometer", Metrologia, 52, 654 (2015).

[5] Y. Bao, F. Zhou, T.W. LeBrun, and J.J. Gorman, "Concave Silicon Micromirrors for Stable Hemispherical Optical Microcavities", Optics Express, 25, 15493 (2017).

[6] Y. Bao, F. Zhou, T.W. LeBrun, and J.J. Gorman, "A Photonic MEMS Accelerometer with a Low-Finesse Hemispherical Microcavity Readout", Int. Conf. Optical MEMS and Nanophotonics, Santa Fe, NM, 111 (2017).

\section{CONTACT}

"J. Gorman, gorman@nist.gov, T. LeBrun, thomas.lebrun@nist.gov 